\title{
Exact Ground States of the Extended Hubbard Model on the Kagomé lattice
}

\author{
Masaaki Nakamura ${ }^{1}$ and Satoshi Nishimoto ${ }^{2,3}$ \\ 1 Department of Physics, Ehime University Bunkyo-cho 2-5, Matsuyama, Ehime 790-8577, Japan, \\ 2 Department of Physics, Technical University Dresden, 01069 Dresden, Germany, \\ 3 Institute for Theoretical Solid State Physics, IFW Dresden, 01171 Dresden, Germany \\ Received: date / Revised version: date
}

\begin{abstract}
We discuss the exact plaquette-ordered ground states of the generalized Hubbard model on the Kagomé lattice for several fillings, by constructing the Hamiltonian as a sum of products of projection operators for up and down spin sectors. The obtained exact ground states are interpreted as Néel ordered states on the bond-located electrons. We determine several parameter regions of the exact ground states, and calculate the entanglement entropy. We examine the above results by numerical calculations based on exact diagonalization and density-matrix renormalization group methods.
\end{abstract}

PACS. 71.10.Fd Lattice fermion models (Hubbard model, etc.) - 71.10.Hf Non-Fermi-liquid ground states, electron phase diagrams and phase transitions in model systems - 75.10.-b General theory and models of magnetic ordering - 71.23. An Theories and models; localized states

\section{Introduction}

The Hubbard model is one of the generic models to describe strongly correlated electron systems [1. This model has played important roles to study magnetism and superconductivity. However, in spite of its simplicity, it is difficult to solve this model exactly except for one dimension or some special cases. On the other hand, extended versions of the Hubbard model have also been studied. The on-site repulsion of the Hubbard model is due to the matrix elements of the Coulomb interaction corresponding to the on-site Wannier states, and other matrix elements are neglected. Therefore, it is worth considering the effects of these neglected terms as site-off-diagonal interactions [2]. For these generalized models, exact results for ferromagnetism and superconducting states have been discussed [3. 4, 5, 6, 7, 8, 9, 10 .

In addition to those, a different type of exact ground state has been discussed for a one-dimensional system, which is called "bond Néel" (BN) state [1, 12,13, by the projection operator method 14,15 for multicomponent systems 16. The BN state is regarded as a Néel ordered state of bond-located spins. Furthermore, the concept of the $\mathrm{BN}$ state in one dimension was extended to higher dimensional systems introducing plaquette states in corner sharing lattices such as the Kagomé lattice 17. In this paper, we extend this argument for the Kagomé Hubbard model at $1 / 3$ filling to several fillings and give numerical verification based on exact diagonalization and densitymatrix renormalization group (DMRG) [18 techniques. We also calculate the entanglement entropy (EE) exactly.
This paper is organized as follows: In Sec. 2, we review the method to construct Hamiltonians with exact ground states in multicomponent systems. In Sec. 3, we review the application of this method to the one-dimensional model discussed in Ref. 11, In Sec. 4, we apply the analysis to the Kagomé lattice. In addition to the exact result at $1 / 3$ filling obtained in Ref. 17, we also discuss the results at $2 / 3$-filling and at half-filling. The exact ground states are numerically confirmed using the exact diagonalization and DMRG methods. In Sec. 5. we calculate the entanglement entropy. Finally, we give summary and discussion of the results.

\section{Construction of the Hamiltonian}

The method to construct a Hamiltonian with an exact ground state is the following way 16 . First, we consider a Hamiltonian given by a sum of products of projection operators

$$
\mathcal{H}=\sum_{\alpha} h_{\alpha}, \quad h_{\alpha}=\sum_{\mu, \nu} \lambda_{\mu \nu} R_{\alpha \uparrow}^{(\mu)} R_{\alpha \downarrow}^{(\nu)}, \quad \lambda_{\mu \nu} \geq 0,
$$

where $\alpha$ denotes the position of one of the unit plaquettes that cover the lattice. $R_{\alpha \sigma}^{(\mu)}$ is an operator whose expectation value is positive semidefinite $\left\langle R_{\alpha \sigma}^{(\mu)}\right\rangle \geq 0$. This condition is realized, if $R_{\alpha \sigma}^{(\mu)}$ is given by a product of an operator and its Hermitian conjugate. Then the expectation value of the Hamiltonian is also positive semidefinite $\langle\mathcal{H}\rangle \geq 0$. 
(a) $1 \mathrm{D}$ chain

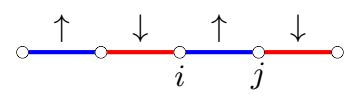

(b)Kagomé

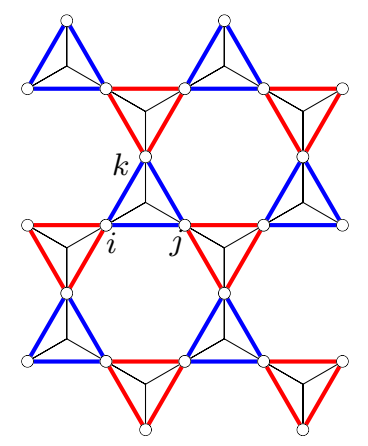

Fig. 1. Examples of lattice structures where generalized Hubbard models with exact plaquette-ordered ground states can be constructed: (a) the one-dimensional chain and (b) the Kagomé lattice. The blue and the red plaquettes denote those belong to the groups $\mathcal{A}$ and $\mathcal{B}$, respectively.

Next, we introduce a trial wave function given by a direct product of up and down spin sectors,

$$
|\Psi(\mathcal{A}, \mathcal{B})\rangle=\left|\Phi_{\uparrow}(\mathcal{A})\right\rangle \otimes\left|\Phi_{\downarrow}(\mathcal{B})\right\rangle
$$

where $\mathcal{A}$ and $\mathcal{B}$ denote two groups of plaquettes that cover the lattice satisfying $\mathcal{A} \cup \mathcal{B}=\{$ all lattice sites $\}$. We require that the projection operators have the following conditions,

$$
R_{\alpha \uparrow}^{(\mu)}\left|\Phi_{\uparrow}(\mathcal{A})\right\rangle=R_{\beta \downarrow}^{(\mu)}\left|\Phi_{\downarrow}(\mathcal{B})\right\rangle=0,
$$

where $\alpha \in \mathcal{A}$ and $\beta \in \mathcal{B}$. Therefore, even if we have

$$
R_{\beta \uparrow}^{(\mu)}\left|\Phi_{\uparrow}(\mathcal{A})\right\rangle \neq 0, \quad R_{\alpha \downarrow}^{(\mu)}\left|\Phi_{\downarrow}(\mathcal{B})\right\rangle \neq 0,
$$

the eigenvalue of the Hamiltonian for $|\Psi(\mathcal{A}, \mathcal{B})\rangle$ is always zero. Then, the lower bound and the upper bound of the energy are coincide, so that $|\Psi(\mathcal{A}, \mathcal{B})\rangle$ turns out to be one of the exact ground state of this system.

The above argument can be satisfied in corner sharing lattices with the bipartite structure. The simplest examples is the one-dimensional (1D) lattice, where the unit plaquette is one bond. In two dimension (2D), the Kagomé lattice can be covered by two colored triangles alternatively, as illustrated in Fig. 1. These states can be regarded as the Néel ordering on the dual lattice (i.e. the honeycomb lattice for the Kagomé lattice). In three dimension, the Pyrochlore lattice satisfies these conditions. If the system has a time-reversal symmetry, its ground state has two-fold degeneracy.

\section{1D model}

We consider the 1D generalized Hubbard model at halffilling and zero-magnetic field, given by $\mathcal{H}=\sum_{i \sigma} h_{i, i+1, \sigma}$ with the local bond Hamiltonian,

$$
\begin{aligned}
& h_{i j \sigma}=-t T_{i j \sigma}+\frac{U}{2 z}\left(n_{i \sigma} n_{i \bar{\sigma}}+n_{j \sigma} n_{j \bar{\sigma}}\right) \\
& +V_{\|} n_{i \sigma} n_{j \sigma}+V_{\perp} n_{i \sigma} n_{j \bar{\sigma}} \\
& +X T_{i j \sigma}\left(n_{i \bar{\sigma}}+n_{j \bar{\sigma}}\right)+\frac{W}{2} \sum_{\sigma^{\prime}} T_{i j \sigma} T_{i j \sigma^{\prime}}
\end{aligned}
$$

where $\bar{\sigma}$ is the opposite spin of $\sigma, z=1$ for the present 1D case, and periodic boundary conditions are assumed. We have defined the hopping and the density operators as $T_{i j \sigma} \equiv c_{i \sigma}^{\dagger} c_{j \sigma}+$ H.c., $n_{i \sigma} \equiv c_{i \sigma}^{\dagger} c_{i \sigma}$. Note that the bondbond interaction $(W)$ term can be rewritten as

$$
-2 W\left(\boldsymbol{S}_{i} \cdot \boldsymbol{S}_{j}+\boldsymbol{\eta}_{i} \cdot \boldsymbol{\eta}_{j}-\frac{1}{4}\right)
$$

where $\boldsymbol{S}_{i}$ and $\boldsymbol{\eta}_{i}$ are the spin and the pseudo spin operators, respectively. The components of the pseudo spin operator are defined by

$\eta_{i}^{+} \equiv(-1)^{i} c_{i \uparrow}^{\dagger} c_{i \downarrow}^{\dagger}, \quad \eta_{i}^{-} \equiv(-1)^{i} c_{i \downarrow} c_{i \uparrow}, \quad \eta_{i}^{z} \equiv \frac{1}{2}\left(n_{i \uparrow}+n_{i \downarrow}-1\right)$.

Now, we introduce the bonding and the anti-bonding operators,

$$
A_{i j \sigma}^{\dagger}=\frac{1}{\sqrt{2}}\left(c_{i \sigma}^{\dagger}+c_{j \sigma}^{\dagger}\right), \quad B_{i j \sigma}^{\dagger}=\frac{1}{\sqrt{2}}\left(c_{i \sigma}^{\dagger}-c_{j \sigma}^{\dagger}\right) .
$$

The two electron states are given by $B_{i j \sigma}^{\dagger} A_{i j \sigma}^{\dagger}=c_{i \sigma}^{\dagger} c_{j \sigma}^{\dagger}$. These operators on the same bond satisfy the anticommutation relations:

$$
\left\{A_{i j \sigma}, A_{i j \sigma^{\prime}}^{\dagger}\right\}=\left\{B_{i j \sigma}, B_{i j \sigma^{\prime}}^{\dagger}\right\}=\delta_{\sigma \sigma^{\prime}}, \quad \text { otherwise }=0 .
$$

The density operators for the bond operators are given as

$$
\begin{aligned}
& n_{A \sigma} \equiv A_{i j \sigma}^{\dagger} A_{i j \sigma}=\frac{1}{2}\left(n_{i \sigma}+n_{j \sigma}+T_{i j \sigma}\right), \\
& n_{B \sigma} \equiv B_{i j \sigma}^{\dagger} B_{i j \sigma}=\frac{1}{2}\left(n_{i \sigma}+n_{j \sigma}-T_{i j \sigma}\right) .
\end{aligned}
$$

Since we restrict our attention only on the neighboring two sites $i, j$, we drop these indices from the operators defined above.

As a trial state, we consider the following wave function,

$$
\left|\Psi_{\sigma}\right\rangle \equiv A_{12 \sigma}^{\dagger} A_{23 \bar{\sigma}}^{\dagger} \cdots A_{L-1, L \sigma}^{\dagger} A_{L, 1 \bar{\sigma}}^{\dagger}|0\rangle
$$

where $|0\rangle$ denotes a vacuum and $L$ is the number of sites. This state is regarded as a Néel ordering of the bondlocated spins, so that we call this bond Néel $(\mathrm{BN})$ state. There is two-fold degeneracy given by $\left|\Psi_{\uparrow}\right\rangle$ and $\left|\Psi_{\downarrow}\right\rangle$. In order to construct a model with the exact ground state, the local Hamiltonian $h_{i j}=\sum_{\sigma} h_{i j \sigma}$ should be decomposed by the projection operators $1-n_{A \sigma}$ and $n_{B \sigma}$ in the following form,

$$
\begin{aligned}
& h_{i j}-\varepsilon_{0}=\lambda_{\bar{A} \bar{A}}\left(1-n_{A \uparrow}\right)\left(1-n_{A \downarrow}\right)+\lambda_{B B} n_{B \uparrow} n_{B \downarrow} \\
& \quad+\lambda_{\bar{A} B}\left\{\left(1-n_{A \uparrow}\right) n_{B \downarrow}+n_{B \uparrow}\left(1-n_{A \downarrow}\right)\right\},
\end{aligned}
$$




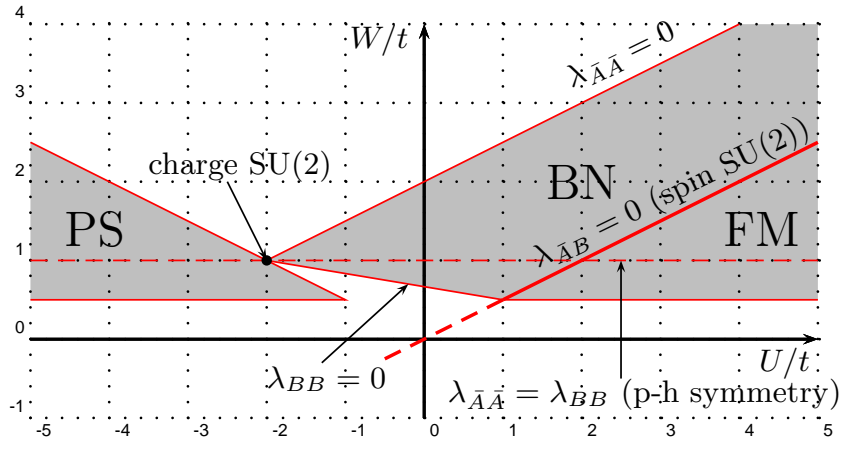

Fig. 2. Phase diagram of the generalized Hubbard chain (5) in the $U / 2 t-W / t$ parameter space with $t>0$ [11,13. The parameters are set as $X=t-W, V_{\|}=W$ and $V_{\perp}=U / 2$. The shaded regions labeled by BN, FM and PS denote bond-Néel, ferromagnetic and phase-separated states, respectively.

where $\varepsilon_{0}$ is the ground-state energy per bond. According to the argument given in Sec. 2, for the BN ground state, the parameters should be chosen as

$$
\lambda_{\bar{A} \bar{A}}, \quad \lambda_{\bar{A} B}, \quad \lambda_{B B} \geq 0 .
$$

Comparing Eqs. (5) and (12) (see Appendix (A), the relations among the parameters are obtained as

$$
V_{\perp}=\frac{U}{2}, \quad V_{\|}=W, \quad X=t-W .
$$

The coefficients in Eq. (12) are identified as follows,

$$
\begin{aligned}
\lambda_{\bar{A} \bar{A}} & =\frac{U}{2}-W+2 t, \\
\lambda_{\bar{A} B} & =-\frac{U}{2}+W, \\
\lambda_{B B} & =\frac{U}{2}+3 W-2 t, \\
\varepsilon_{0} & =\frac{U}{2} .
\end{aligned}
$$

From Eqs. (13), (15), (16) and (17), we obtain the parameter space of the exact BN ground state as shown in Fig. 2 Note that the BN state appears only for $t>0$ region.

The property of the BN state can be investigated based on the matrix-product method. According to Ref. 11, both charge-charge and spin-spin correlation functions vanish except for those of the nearest sites which indicates the existence of the charge and the spin gaps. On the other hand, the bond-located spin correlation exhibits a long range order. We can also calculate elementally excitation spectrum using the matrix-product method as a variational approach [13].

In the present one-dimensional model at half-filling, we can discuss not only the BN state but also the ferromagnetic (FM) and the phase-separated (PS) states. The last term of Eq. (12) stabilizes the fully polarized FM state for $\lambda_{\bar{A} B}<0$. Similarly, the PS state where the system is separated into a domain of doubly occupied sites and a vacuum, is stabilized when $\lambda_{\bar{A} \bar{A}}+\lambda_{B B}<0$, neglecting the surface energy. As shown in Fig. 2, the FM and the PS states appear in the $U / 2 t-W / t$ parameter space symmetrically in the positive- and in the negative- $U$ regions, respectively. This is consistent with the fact that the $W$ term is the ferromagnetic exchange interactions of the spins and the pseudo spins (6), and the PS state is regarded as the FM state of the pseudo-spin space. The condition $W / t \geq 1 / 2$ for the FM and the PS phases is not clearly obtained in the present argument. To obtain this condition, we need to introduce three-types of $R$ operators $[13$.

The phase boundary of the $\mathrm{BN}$ and the FM states $\lambda_{\bar{A} B}=0$ corresponds to the $\mathrm{SU}(2)$ symmetry in the spins $V_{\|}=V_{\perp}$, so that the ground state is highly degenerate. The system undergoes a first-order phase transition at this level-crossing point. When $W / t=1(X=0)$, the system has the particle-hole symmetry. At $(U / 2 t, W / t)=(-1,1)$, the system has the $\mathrm{SU}(2)$ symmetry in the pseudo-spin space, so that the $\mathrm{BN}$, the PS and the $\eta$-paring states are degenerate. The other lines which separate shaded and non-shaded regions in Fig. 2 do not necessarily mean phase boundaries.

Therefore, to confirm the BN and FM states and to explore the phase boundaries, we calculate the ground-state energy by the numerical methods. In Fig. 3)(a) the numerical results of the ground-state energy at $U=2 t$ are plotted as a function of $W / t$, where the periodic boundary conditions are applied. We obtain numerically the BN groundstate energy $\varepsilon_{0}=U / 2 \equiv \varepsilon_{0}(\mathrm{BN})$ for $W \geq U / 2$ and the FM ground-state energy $\varepsilon_{0}=2 W \equiv \varepsilon_{0}(\mathrm{FM})$ for $W \leq U / 2$. Thus, the BN-FM phase boundary coincides the analytical result $W=U / 2$. The ground-state energy deviates from $\varepsilon_{0}=\varepsilon_{0}(\mathrm{BN})$ at some larger $W / t\left(\equiv W_{c} / t\right)$, which corresponds to the upper bound of the $\mathrm{BN}$ phase and is detected as a level crossing in the present finite- $L$ calculations. As seen in Fig. 3(a), the level-crossing point depends on the system length because the BN state is more overstabilized in smaller- $L$ systems under the periodic boundary conditions. Accordingly, the level-crossing point is shifted to lower $W / t$ with increasing the system length $L$. We perform a finite-size scaling of the level-crossing point using $L=26-50$ periodic systems in Fig. 3(b). Although the data points oscillate and a fine fitting is not easy, the least-square linear fitting gives $W_{c} / t=6.37$ in the thermodynamic limit. This may mean the upper bound of the $\mathrm{BN}$ phase is fairly extended to $W / t=6.37$ in comparison to the analytical value $W / t=3$ in Fig. 2 ,

The above overstabilization of the BN state can be avoided if we apply the open boundary conditions. It enables us to pick up the real ground state and to calculate the energy more definitely for a given $L$. The extrapolated ground-state energy to the thermodynamic limit, using $L=48-240$ open systems, is plotted in Fig. 33(c). We find that the ground-state energy begins to deviate from $\varepsilon_{0}=\varepsilon_{0}(\mathrm{BN})$ at $W / t=6.40$. This value agrees very well with that obtained with the periodic systems $(W / t=6.37)$. 
(a)

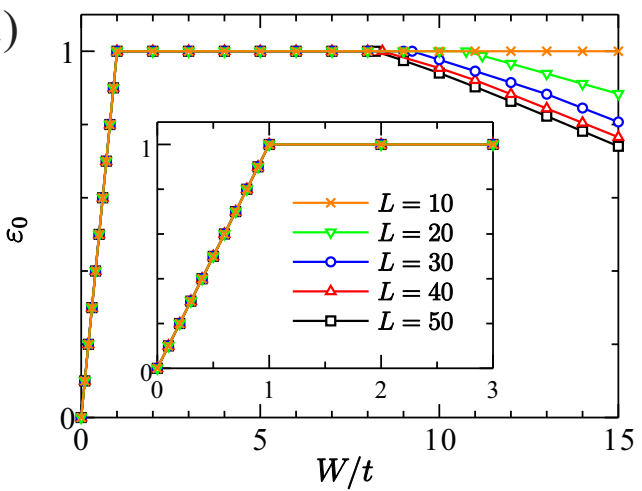

(b)

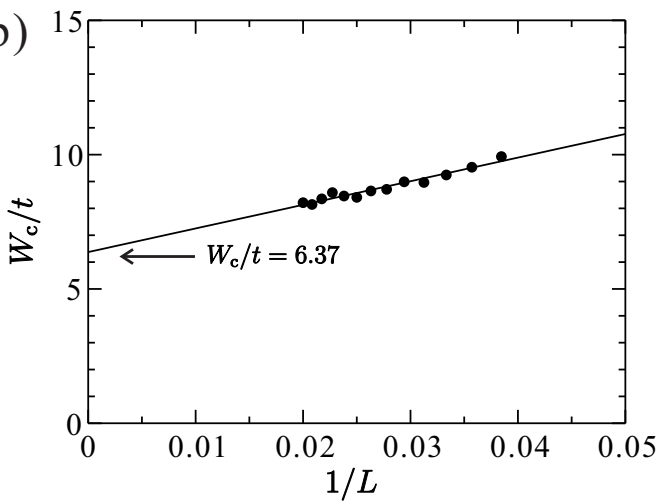

(c)

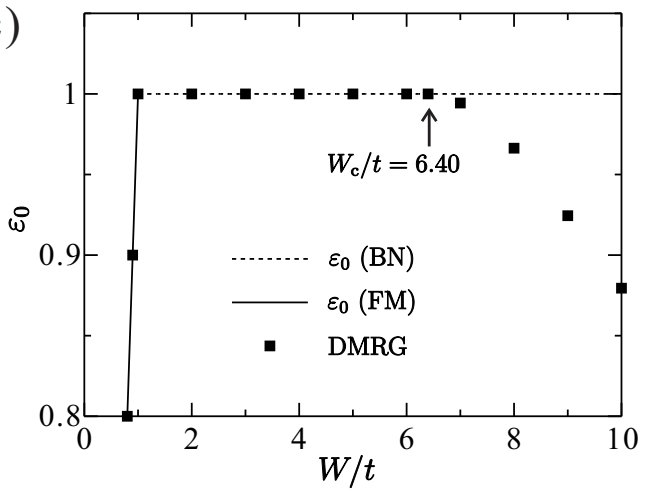

Fig. 3. (a) Ground-state energy per site as a function of $W / t$ for the $1 \mathrm{D}$ model at $U / t=2$, obtained by the exact diagonalization and DMRG with finite- $L$ chains under periodic boundary conditions. Inset: enlarged figure around the lower level crossing. (b) Finite-size scaling analysis of the level-crossing points for the upper bound of the BN phase. (c) Ground-state energy per site as a function of $W / t$ for the $1 \mathrm{D}$ model at $U / t=2$ in the thermodynamic limit, which is obtained using DMRG data with $L=48-240$ chains under the open boundary conditions.

\section{Kagomé lattice}

We consider the generalized Hubbard model on the Kagomé lattice at $1 / 3$-filling with zero-magnetic field. In order to obtain an exact ground state, we need to include three site terms $\left(X^{\prime}, W^{\prime}\right.$ terms). The Hamiltonian is given by $\mathcal{H}=\sum_{\langle i j k\rangle \sigma} h_{i j k \sigma}$, where the summation $\langle i j k\rangle$ is taken in

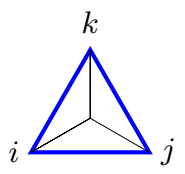

$\left|A_{i j k \sigma}\right\rangle$

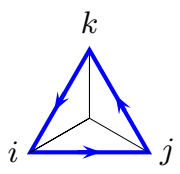

$\left|B_{i j k \sigma}\right\rangle$

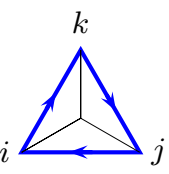

$\left|C_{i j k \sigma}\right\rangle$
Fig. 4. Three bases for the unit trimer of the Kagomé lattice.

each unit trimer as shown in Fig. 1

$$
\begin{aligned}
& h_{i j k \sigma}=h_{i j \sigma}+h_{j k \sigma}+h_{k i \sigma} \\
& +W^{\prime}\left(T_{i j \sigma} T_{j k \bar{\sigma}}+T_{j k \sigma} T_{k i \bar{\sigma}}+T_{k i \sigma} T_{i j \bar{\sigma}}\right) \\
& +X^{\prime}\left(T_{i j \sigma} n_{k \bar{\sigma}}+T_{j k \sigma} n_{i \bar{\sigma}}+T_{k i \sigma} n_{j \bar{\sigma}}\right),
\end{aligned}
$$

where $h_{i j \sigma}$ is the local bond Hamiltonian (5) with $z=2 . \bar{\sigma}$ denotes the opposite spin of $\sigma$. Now we define the following one-electron plaquette operators (see Fig. (4),

$$
\begin{aligned}
A_{i j k \sigma}^{\dagger} & \equiv \frac{1}{\sqrt{3}}\left(c_{i \sigma}^{\dagger}+c_{j \sigma}^{\dagger}+c_{k \sigma}^{\dagger}\right), \\
B_{i j k \sigma}^{\dagger} & \equiv \frac{1}{\sqrt{3}}\left(c_{i \sigma}^{\dagger}+\omega c_{j \sigma}^{\dagger}+\omega^{2} c_{k \sigma}^{\dagger}\right), \\
C_{i j k \sigma}^{\dagger} & \equiv \frac{1}{\sqrt{3}}\left(c_{i \sigma}^{\dagger}+\omega^{2} c_{j \sigma}^{\dagger}+\omega c_{k \sigma}^{\dagger}\right),
\end{aligned}
$$

where $\omega=\mathrm{e}^{12 \pi / 3}$. These operators on the same plaquette satisfy the anticommutation relations:

$\left\{A_{i j k \sigma}, A_{i j k \sigma^{\prime}}^{\dagger}\right\}=\left\{B_{i j k \sigma}, B_{i j k \sigma^{\prime}}^{\dagger}\right\}=\left\{C_{i j k \sigma}, C_{i j k \sigma^{\prime}}^{\dagger}\right\}=\delta_{\sigma \sigma^{\prime}}$, and otherwise $=0$. Note that $A_{i j k \sigma}^{\dagger}|0\rangle, B_{i j k \sigma}^{\dagger}|0\rangle$, and $C_{i j k \sigma}^{\dagger}|0\rangle$ are chosen as eigen states of density, hopping, and current operators,

$$
\begin{aligned}
N_{i j k \sigma} & \equiv n_{i \sigma}+n_{j \sigma}+n_{k \sigma}, \\
T_{i j k \sigma} & \equiv T_{i j \sigma}+T_{j k \sigma}+T_{k i \sigma}, \\
J_{i j k \sigma} & \equiv J_{i j \sigma}+J_{j k \sigma}+J_{k i \sigma}, \\
J_{i j \sigma} & \equiv 1\left(c_{i \sigma}^{\dagger} c_{j \sigma}-\text { H.c. }\right) .
\end{aligned}
$$

The density operators in terms of the plaquette operators are

$$
\begin{aligned}
& n_{A \sigma}=\frac{1}{3}\left(N_{i j k \sigma}+T_{i j k \sigma}\right), \\
& n_{B \sigma}=\frac{1}{6}\left(2 N_{i j k \sigma}-T_{i j k \sigma}-\sqrt{3} J_{i j k \sigma}\right), \\
& n_{C \sigma}=\frac{1}{6}\left(2 N_{i j k \sigma}-T_{i j k \sigma}+\sqrt{3} J_{i j k \sigma}\right) .
\end{aligned}
$$

Since we restrict our attention only on the three sites $i, j, k$ in a triangle, we drop these indices from the operators defined above.

\subsection{Plaquette-Néel state at $1 / 3$-filling}

Using these relations, the Hamiltonian with the exact ground state is given by the plaquette operators. We consider the following plaquette state at $1 / 3$-filling,

$$
\left|\Psi_{\sigma}\right\rangle \equiv \prod_{\langle i j k\rangle \in \triangle} A_{i j k \sigma}^{\dagger} \prod_{\left\langle i^{\prime} j^{\prime} k^{\prime}\right\rangle \in \nabla} A_{i^{\prime} j^{\prime} k^{\prime} \sigma}^{\dagger}|0\rangle,
$$


where $\langle i j k\rangle\left(\left\langle i^{\prime} j^{\prime} k^{\prime}\right\rangle\right)$ is taken for all triangles of the Kagomé lattice with up (down) direction. As an extention of the BN state, we call this state "plaquette Néel" (PN) state.

In order to make (30) the ground state, the local Hamiltonian for this state is constructed as

$$
\begin{aligned}
& \quad h_{i j k}-\varepsilon_{0}=\lambda_{\bar{A} \bar{A}}\left(1-n_{A \uparrow}\right)\left(1-n_{A \downarrow}\right) \\
& \quad+\lambda_{B B} n_{B \uparrow} n_{B \downarrow}+\lambda_{C C} n_{C \uparrow} n_{C \downarrow} \\
& \quad+\lambda_{\bar{A} B}\left\{\left(1-n_{A \uparrow}\right) n_{B \downarrow}+n_{B \uparrow}\left(1-n_{A \downarrow}\right)\right\} \\
& \quad+\lambda_{\bar{A} C}\left\{\left(1-n_{A \uparrow}\right) n_{C \downarrow}+n_{C \uparrow}\left(1-n_{A \downarrow}\right)\right\} \\
& \quad+\lambda_{B C}\left\{n_{B \uparrow} n_{C \downarrow}+n_{C \uparrow} n_{B \downarrow}\right\} \\
& =\lambda_{\bar{A} \bar{A}} \\
& \quad+\sum_{\sigma}\left\{-\lambda_{\bar{A} \bar{A}} n_{A \sigma}+\lambda_{\bar{A} B} n_{B \sigma}+\lambda_{\bar{A} C} n_{C \sigma}\right\} \\
& \quad+\lambda_{\bar{A} \bar{A}} n_{A \uparrow} n_{A \downarrow}+\lambda_{B B} n_{B \uparrow} n_{B \downarrow}+\lambda_{C C} n_{C \uparrow} n_{C \downarrow} \\
& \quad-\lambda_{\bar{A} B}\left(n_{A \uparrow} n_{B \downarrow}+n_{B \uparrow} n_{A \downarrow}\right) \\
& \quad-\lambda_{\bar{A} C}\left(n_{A \uparrow} n_{C \downarrow}+n_{C \uparrow} n_{A \downarrow}\right) \\
& \quad+\lambda_{B C}\left(n_{B \uparrow} n_{C \downarrow}+n_{C \uparrow} n_{B \downarrow}\right),
\end{aligned}
$$

with positive $\lambda_{\mu \nu}$. Here we consider the case that $\lambda_{B B}=$ $\lambda_{C C}$ and $\lambda_{\bar{A} B}=\lambda_{\bar{A} C}$, assuming the time-reversal symmetry of the Hamiltonian. Then we have

$$
\begin{aligned}
& h_{i j k}-\varepsilon_{0}=\frac{1}{3}\left(\lambda_{\bar{A} \bar{A}}+\lambda_{\bar{A} B}\right) h_{t} \\
& +\frac{1}{9}\left(\lambda_{\bar{A} \bar{A}}-4 \lambda_{\bar{A} B}+4 \lambda_{B B}\right)\left(2 h_{U}+h_{V_{\perp}}\right) \\
& +\frac{1}{9}\left(\lambda_{\bar{A} \bar{A}}+2 \lambda_{\bar{A} B}+\lambda_{B B}\right)\left(h_{V_{\|}}+h_{W}+h_{W^{\prime}}\right) \\
& +\frac{1}{9}\left(\lambda_{\bar{A} \bar{A}}-\lambda_{\bar{A} B}-2 \lambda_{B B}\right)\left(h_{X}+h_{X^{\prime}}\right) \\
& +\frac{1}{9}\left(-4 \lambda_{\bar{A} \bar{A}}+4 \lambda_{\bar{A} B}-\lambda_{B B}\right) \sum_{\sigma} N_{i j k \sigma}+\lambda_{\bar{A} \bar{A}},
\end{aligned}
$$

where $h_{t}, h_{U}, \cdots, h_{X^{\prime}}$ are defined in Appendix A, For $1 / 3$-filling, the density operator and the number of the triangles $N_{\mathrm{tr}}$ is related as $\sum_{\langle i j k\rangle, \sigma} N_{i j k \sigma}=2 N_{\mathrm{tr}}$, and the number of lattice sites is $L=3 N_{\mathrm{tr}} / 2$, so that the groundstate energy per site is identified as

$$
\varepsilon_{0}=\frac{1}{9}\left(\lambda_{\bar{A} \bar{A}}+8 \lambda_{\bar{A} B}-2 \lambda_{B B}\right) .
$$

The coefficients of projection operators are related to the parameters as

$$
\left[\begin{array}{l}
\lambda_{\bar{A} \bar{A}} \\
\lambda_{\bar{A} B} \\
\lambda_{B B}
\end{array}\right]=\left[\begin{array}{ccc}
1 & 4 & 4 \\
-1 & 2 & -1 \\
1 & 1 & -2
\end{array}\right]\left[\begin{array}{c}
U / 2 \\
W \\
X
\end{array}\right] .
$$

Using the condition of the hopping in Eq. (33), we have

$$
\begin{aligned}
& \lambda_{\bar{A} \bar{A}}=\frac{U}{2}-4 W+4 t, \\
& \lambda_{\bar{A} B}=-\frac{U}{2}+4 W-t, \\
& \lambda_{B B}=\frac{U}{2}+5 W-2 t .
\end{aligned}
$$
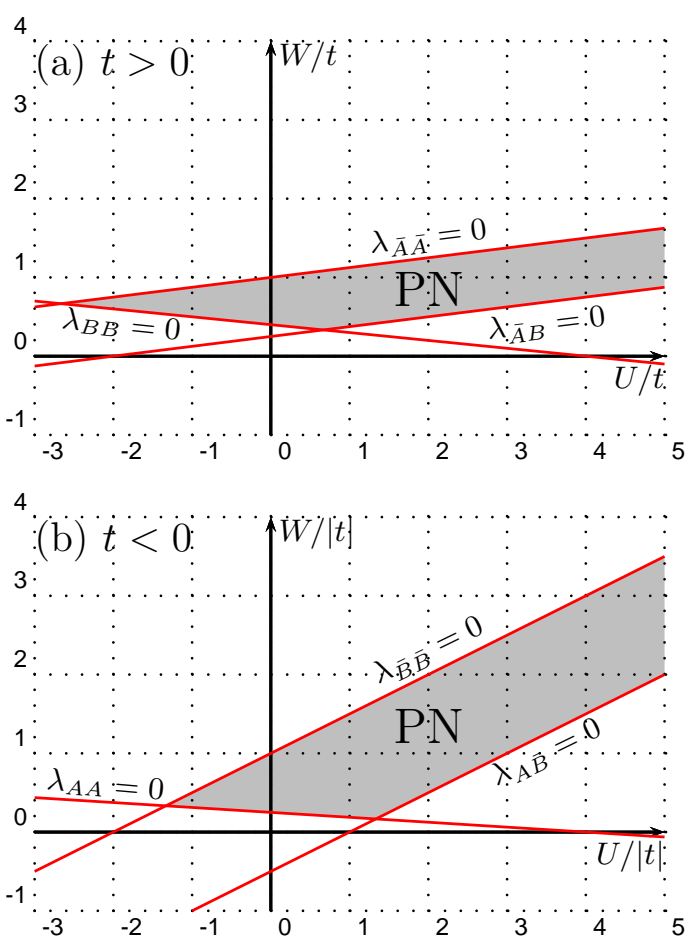

Fig. 5. Phase diagrams of the generalized Hubbard model on the Kagomé lattice, in the $U /|t|-W /|t|$ parameter space for (a) $t>0$ at $1 / 3$-filling [17] and (b) $t<0$ at 2/3-filling, respectively. The shaded regions labeled by PN denote the plaquette Néel state.

Since all these coefficients should be positive, the condition of the exact PN ground state is given as follows,

$$
\begin{gathered}
W \leq \frac{U}{8}+t, \quad W \geq \frac{U}{8}+\frac{t}{4}, \quad W \geq-\frac{U}{10}+\frac{2 t}{5}, \\
V_{\perp}=\frac{U}{2}, \quad V_{\|}=W=W^{\prime}, \quad X=X^{\prime}=t-2 W,
\end{gathered}
$$

and the ground state energy per site is

$$
\varepsilon_{0}=\frac{1}{3}(U-4 W) .
$$

The phase diagram for the exact $\mathrm{PN}$ state is surrounded by three lines given by $\lambda_{\bar{A} \bar{A}}>0, \lambda_{\bar{A} B}>0$, and $\lambda_{B B}>0$, as shown in Fig 5(a).

In Fig. 6, the numerical results of the ground-state energy for the $1 / 3$-filling Kagomé model at $U / t=2$ with $t>0$ are plotted as a function of $W / t$. The energy of the PN state $\varepsilon_{0}=(U-4 W) / 3 \equiv \varepsilon_{0}(\mathrm{PN})$ is subtracted so that a region with $\varepsilon_{0}-\varepsilon_{0}(\mathrm{PN})=0$ corresponds to the PN phase. As seen in Figs. 6(a) and (b), a robust range with $\varepsilon_{0}-\varepsilon_{0}(\mathrm{PN})=0$ exists for all the used clusters. We find a deviation from $\varepsilon_{0}-\varepsilon_{0}(\mathrm{PN})=0$ below a levelcrossing point $W / t=0.5$, which is independent of the cluster shape and size [see insets of Figs. 6(a) and (b)]. This $W / t$ value agrees perfectly with the analytical result of the lower bound of the PN phase, given by $\lambda_{\bar{A} B}=$ 0 . Whereas, the level-crossing point related to the upper bound depends on the cluster. Like in the $1 \mathrm{D}$ BN state, 

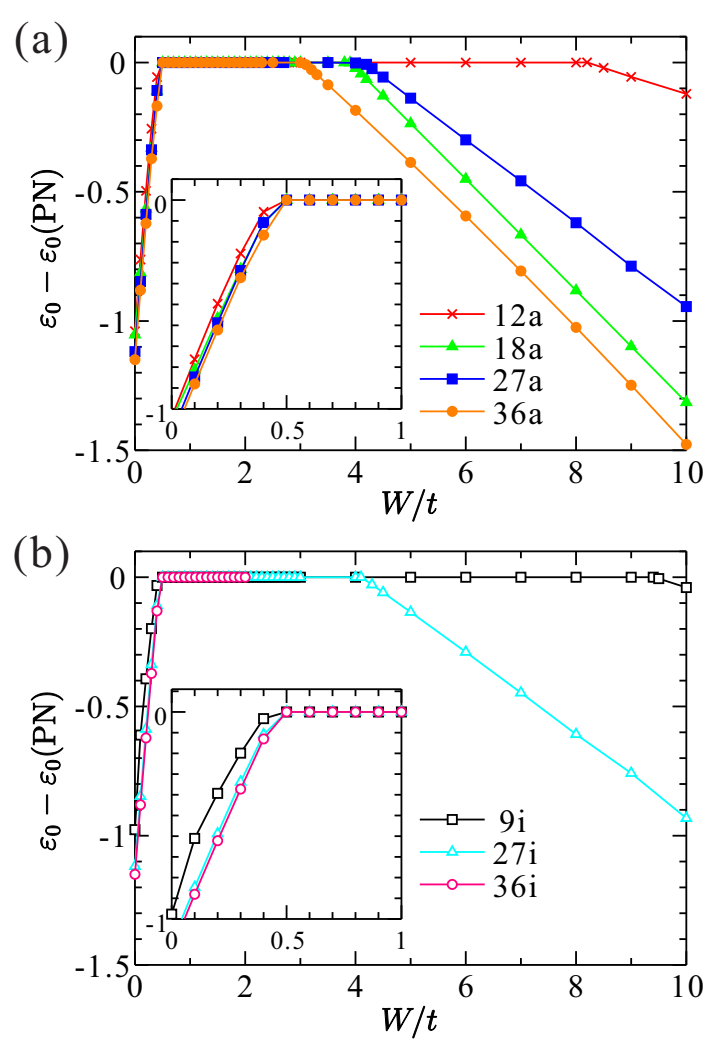

Fig. 6. (a) Ground-state energy per site as a function of $W / t$ for the $1 / 3$-filling Kagomé model with $U / t=2$ and $t>0$, obtained by the numerical methods using (a) anisotropic and (b) isotropic clusters (see Appendix B). The energy of the PN state [Eq.(39)] is subtracted. Insets: enlarged figure around the lower level crossing.

the PN state would be overstabilized with small clusters. However, the data are not sufficient to perform a finite-size scaling analysis and it remains as a future work.

\subsection{Plaquette-Néel state at 2/3-filling}

We consider the following plaquette Néel state at 2/3filling given by

$$
\left|\Psi_{\sigma}\right\rangle \equiv \prod_{\langle i j k\rangle \in \triangle} C_{i j k \sigma}^{\dagger} B_{i j k \sigma}^{\dagger} \prod_{\left\langle i^{\prime} j^{\prime} k^{\prime}\right\rangle \in \nabla} C_{i^{\prime} j^{\prime} k^{\prime} \bar{\sigma}}^{\dagger} B_{i^{\prime} j^{\prime} k^{\prime} \bar{\sigma}}^{\dagger}|0\rangle
$$

where $\langle i j k\rangle\left(\left\langle i^{\prime} j^{\prime} k^{\prime}\right\rangle\right)$ is taken for all triangles of the Kagomé lattice with up (down) direction, and

$$
C_{i j k \sigma}^{\dagger} B_{i j k \sigma}^{\dagger}=\frac{1}{\sqrt{3}}\left(c_{i \sigma}^{\dagger} c_{j \sigma}^{\dagger}+c_{j \sigma}^{\dagger} c_{k \sigma}^{\dagger}+c_{k \sigma}^{\dagger} c_{i \sigma}^{\dagger}\right)
$$

The Hamiltonian for this state is constructed as

$$
\begin{aligned}
& h_{i j k}-\varepsilon_{0}=\lambda_{A A} n_{A \uparrow} n_{A \downarrow} \\
& +\lambda_{\bar{B} \bar{B}}\left(1-n_{B \uparrow}\right)\left(1-n_{B \downarrow}\right)+\lambda_{\bar{C} \bar{C}}\left(1-n_{C \uparrow}\right)\left(1-n_{C \downarrow}\right) \\
& +\lambda_{A \bar{B}}\left\{n_{A \uparrow}\left(1-n_{B \downarrow}\right)+\left(1-n_{B \uparrow}\right) n_{A \downarrow}\right\} \\
& +\lambda_{A \bar{C}}\left\{n_{A \uparrow}\left(1-n_{C \downarrow}\right)+\left(1-n_{C \uparrow}\right) n_{A \downarrow}\right\} \\
& +\lambda_{\bar{B} \bar{C}}\left\{\left(1-n_{B \uparrow}\right)\left(1-n_{C \downarrow}\right)+\left(1-n_{C \uparrow}\right)\left(1-n_{B \downarrow}\right)\right\} \\
& =\lambda_{\bar{B} \bar{B}}+\lambda_{\bar{C} \bar{C}}+2 \lambda_{\bar{B} \bar{C}} \\
& \quad+\sum_{\sigma}\left\{\left(\lambda_{A \bar{B}}+\lambda_{A \bar{C}}\right) n_{A \sigma}-\left(\lambda_{\bar{B} \bar{B}}+\lambda_{\bar{B} \bar{C}}\right) n_{B \sigma}\right. \\
& \left.\quad-\left(\lambda_{\bar{C} \bar{C}}+\lambda_{\bar{B} \bar{C}}\right) n_{C \sigma}\right\} \\
& +\lambda_{A A} n_{A \uparrow} n_{A \downarrow}+\lambda_{\bar{B} \bar{B}} n_{B \uparrow} n_{B \downarrow}+\lambda_{\bar{C} \bar{C}} n_{C \uparrow} n_{C \downarrow} \\
& -\lambda_{A \bar{B}}\left(n_{A \uparrow} n_{B \downarrow}+n_{B \uparrow} n_{A \downarrow}\right) \\
& -\lambda_{A \bar{C}}\left(n_{A \uparrow} n_{C \downarrow}+n_{C \uparrow} n_{A \downarrow}\right) \\
& +\lambda_{\bar{B} \bar{C}}\left(n_{B \uparrow} n_{C \downarrow}+n_{C \uparrow} n_{B \downarrow}\right) .
\end{aligned}
$$

For $\lambda_{\bar{B} \bar{B}}=\lambda_{\bar{B} \bar{C}}=\lambda_{\bar{C} \bar{C}}$, and $\lambda_{A \bar{B}}=\lambda_{A \bar{C}}$, assuming the time-reversal symmetry of the Hamiltonian, we have

$$
\begin{aligned}
& h_{i j k}-\varepsilon_{0}=\frac{2}{3}\left(-\lambda_{A \bar{B}}-\lambda_{\bar{B} \bar{B}}\right) h_{t} \\
& +\frac{1}{9}\left(\lambda_{A A}-4 \lambda_{A \bar{B}}+4 \lambda_{\bar{B} \bar{B}}\right)\left(2 h_{U}+h_{V_{\perp}}\right) \\
& +\frac{1}{9}\left(\lambda_{A A}+2 \lambda_{A \bar{B}}+\lambda_{\bar{B} \bar{B}}\right)\left(h_{V_{\|}}+h_{W}+h_{W^{\prime}}\right) \\
& +\frac{1}{9}\left(\lambda_{A A}-\lambda_{A \bar{B}}-2 \lambda_{\bar{B} \bar{B}}\right)\left(h_{X}+h_{X^{\prime}}\right) \\
& +\frac{1}{9}\left(-\lambda_{A A}+4 \lambda_{A \bar{B}}-13 \lambda_{\bar{B} \bar{B}}\right) \sum_{\sigma} N_{i j k \sigma}+4 \lambda_{\bar{B} \bar{B}} .
\end{aligned}
$$

For 2/3-filling, the density operator and the number of the triangles $N_{\mathrm{tr}}$ is related as $\sum_{\langle i j k\rangle, \sigma} N_{i j k \sigma}=4 N_{\mathrm{tr}}$, and the number of lattice sites is $L=3 N_{\mathrm{tr}} / 2$, so that the groundstate energy per site is identified as

$$
\varepsilon_{0}=\frac{8}{27}\left(\lambda_{A A}-4 \lambda_{A \bar{B}}+4 \lambda_{\bar{B} \bar{B}}\right) .
$$

Since the relation between $\left(\lambda_{A A}, \lambda_{A \bar{B}}, \lambda_{\bar{B} \bar{B}}\right)$ and $(U, W, X)$ is given by the same matrix as that of (35), we identify the coefficients of the projection operators, using the condition for the hopping in Eq. (44), as

$$
\begin{aligned}
& \lambda_{A A}=\frac{U}{2}+8 W+2 t, \\
& \lambda_{A \bar{B}}=-\frac{U}{2}+W-\frac{t}{2}, \\
& \lambda_{\bar{B} \bar{B}}=\frac{U}{2}-W-t .
\end{aligned}
$$

Thus the condition of the exact PN ground state is given as follows,

$$
\begin{gathered}
W \geq-\frac{U}{16}-\frac{t}{4}, \quad W \geq \frac{U}{2}+\frac{t}{2}, \quad W \leq \frac{U}{2}-t, \\
V_{\perp}=\frac{U}{2}, \quad V_{\|}=W=W^{\prime}, \quad X=X^{\prime}=\frac{t}{2}+W .
\end{gathered}
$$



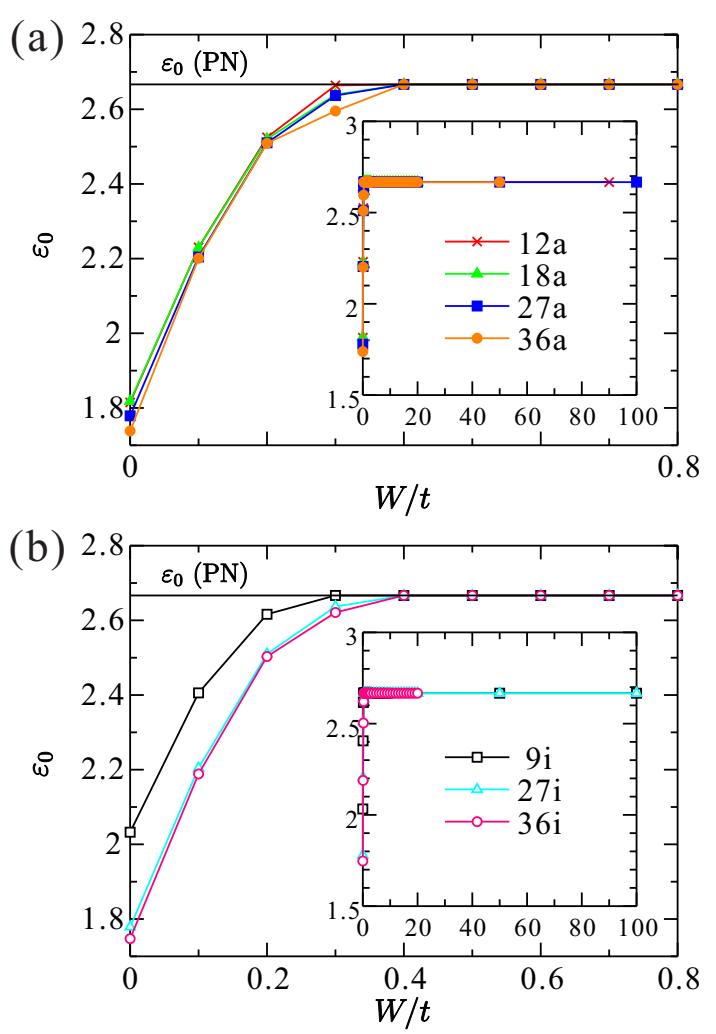

Fig. 7. (a) Ground-state energy per site as a function of $W /|t|$ for the 2/3-filling Kagomé model with $U /|t|=2$ and $t<0$, obtained by the numerical methods using (a) anisotropic and (b) isotropic clusters (see Appendix B). Insets: similar figures for a wider range of $W /|t|$.

The ground state energy per site is

$$
\varepsilon_{0}=\frac{4}{3} U
$$

The phase diagram for the exact plaquette Néel state is surrounded by three boundaries given by $\lambda_{A A}>0, \lambda_{A \bar{B}}>$ 0 , and $\lambda_{\bar{B} \bar{B}}>0$, as shown in Fig 5 (b).

The numerical results of the ground-state energy for the 2/3-filling Kagomé model at $U /|t|=2$ with $t<0$ are plotted as a function of $W /|t|$ in Fig. (7. We find that the system has the PN state energy $\varepsilon_{0}(\mathrm{PN})=4 U / 3$ in a wide range of $W /|t|$. The energy deviation from $\varepsilon_{0}=$ $\varepsilon_{0}(\mathrm{PN})$, indicating a transition to another phase, is clearly seen. Although the level crossing is not very sharp, we can approximately estimate the transition point $W /|t| \sim 0.4$ for all the used clusters. This value is close but subtly smaller than the analytical result of the lower bound of the PN phase $W /|t|=U /(2|t|)-1 / 2=0.5$ given by $\lambda_{A \bar{B}}=0$. Let us then turn to the upper bound of the PN phase. It may be more puzzling. Differently from the case of $1 / 3-$ filling Kagomé lattice with $t>0$, the PN state seems to maintain as the ground state up to $W /|t|=100$ in the present calculations with periodic clusters. To resolve this issue, further calculations are required.

\subsection{Ferromagnetism at $1 / 2$-filling}

We consider a ferromagnetic (FM) state at half-filling where each triangle is occupied by three particles with the same spin,

$$
\left|\Psi_{\sigma}\right\rangle \equiv \prod_{\langle i j k\rangle \in \triangle} C_{i j k \sigma}^{\dagger} B_{i j k \sigma}^{\dagger} A_{i j k \sigma}^{\dagger}|0\rangle
$$

where

$$
C_{i j k \sigma}^{\dagger} B_{i j k \sigma}^{\dagger} A_{i j k \sigma}^{\dagger}=1 c_{i \sigma}^{\dagger} c_{j \sigma}^{\dagger} c_{k \sigma}^{\dagger} .
$$

The Hamiltonian for this state is constructed as

$$
\begin{aligned}
& h_{i j k}-\varepsilon_{0}=\lambda_{\bar{A} \bar{A}}\left(1-n_{A \uparrow}\right)\left(1-n_{A \downarrow}\right) \\
& +\lambda_{\bar{B} \bar{B}}\left(1-n_{B \uparrow}\right)\left(1-n_{B \downarrow}\right) \\
& +\lambda_{\bar{C} \bar{C}}\left(1-n_{C \uparrow}\right)\left(1-n_{C \downarrow}\right) \\
& +\lambda_{\bar{A} \bar{B}}\left\{\left(1-n_{A \uparrow}\right)\left(1-n_{B \downarrow}\right)+\left(1-n_{B \uparrow}\right)\left(1-n_{A \downarrow}\right)\right\} \\
& +\lambda_{\bar{A} \bar{C}}\left\{\left(1-n_{A \uparrow}\right)\left(1-n_{C \downarrow}\right)+\left(1-n_{C \uparrow}\right)\left(1-n_{A \downarrow}\right)\right\} \\
& +\lambda_{\bar{B} \bar{C}}\left\{\left(1-n_{B \uparrow}\right)\left(1-n_{C \downarrow}\right)+\left(1-n_{C \uparrow}\right)\left(1-n_{B \downarrow}\right)\right\} \\
& =\lambda_{\bar{A} \bar{A}}+\lambda_{\bar{B} \bar{B}}+\lambda_{\bar{C} \bar{C}}+2\left(\lambda_{\bar{A} \bar{B}}+\lambda_{\bar{B} \bar{C}}+\lambda_{\bar{A} \bar{C}}\right) \\
& \quad+\sum_{\sigma}\left\{-\left(\lambda_{\bar{A} \bar{A}}+\lambda_{\bar{A} \bar{B}}+\lambda_{\bar{A} \bar{C}}\right) n_{A \sigma}\right. \\
& \quad-\left(\lambda_{\bar{B} \bar{B}}+\lambda_{\bar{A} \bar{B}}+\lambda_{\bar{B} \bar{C}}\right) n_{B \sigma} \\
& \left.\quad-\left(\lambda_{\bar{C} \bar{C}}+\lambda_{\bar{A} \bar{C}}+\lambda_{\bar{B} \bar{C}}\right) n_{C \sigma}\right\} \\
& \quad+\lambda_{\bar{A} \bar{A}} n_{A \uparrow} n_{A \downarrow}+\lambda_{\bar{B} \bar{B}} n_{B \uparrow} n_{B \downarrow}+\lambda_{\bar{C} \bar{C}} n_{C \uparrow} n_{C \downarrow} \\
& \quad+\lambda_{\bar{A} \bar{B}}\left(n_{A \uparrow} n_{B \downarrow}+n_{B \uparrow} n_{A \downarrow}\right) \\
& \quad+\lambda_{\bar{A} \bar{C}}\left(n_{A \uparrow} n_{C \downarrow}+n_{C \uparrow} n_{A \downarrow}\right) \\
& \quad+\lambda_{\bar{B} \bar{C}}\left(n_{B \uparrow} n_{C \downarrow}+n_{C \uparrow} n_{B \downarrow}\right) .
\end{aligned}
$$

Under the time-reversal symmetry $\lambda_{\bar{B} \bar{B}}=\lambda_{\bar{B} \bar{C}}=\lambda_{\bar{C} \bar{C}}$, and $\lambda_{\bar{A} \bar{B}}=\lambda_{\bar{A} \bar{C}}$, we have

$$
\begin{aligned}
& h_{i j k}-\varepsilon_{0}=\frac{1}{3}\left(\lambda_{\bar{A} \bar{A}}+\lambda_{\bar{A} \bar{B}}-2 \lambda_{\bar{B} \bar{B}}\right) h_{t} \\
& +\frac{1}{9}\left(\lambda_{\bar{A} \bar{A}}+4 \lambda_{\bar{A} \bar{B}}+4 \lambda_{\bar{B} \bar{B}}\right)\left(2 h_{U}+h_{V_{\perp}}\right) \\
& +\frac{1}{9}\left(\lambda_{\bar{A} \bar{A}}-2 \lambda_{\bar{A} \bar{B}}+\lambda_{\bar{B} \bar{B}}\right)\left(h_{V_{\|}}+h_{W}+h_{W^{\prime}}\right) \\
& +\frac{1}{9}\left(\lambda_{\bar{A} \bar{A}}+\lambda_{\bar{A} \bar{B}}-2 \lambda_{\bar{B} \bar{B}}\right)\left(h_{X}+h_{X^{\prime}}\right) \\
& -\frac{1}{9}\left(4 \lambda_{\bar{A} \bar{A}}+10 \lambda_{\bar{A} \bar{B}}+13 \lambda_{\bar{B} \bar{B}}\right) \sum_{\sigma} N_{i j k \sigma} \\
& +\lambda_{\bar{A} \bar{A}}+4 \lambda_{\bar{A} \bar{B}}+4 \lambda_{\bar{B} \bar{B}} .
\end{aligned}
$$

At half-filling, the number of the triangle $N_{\mathrm{tr}}$ is related as $\sum_{\langle i j k\rangle, \sigma} N_{i j k \sigma}=3 N_{\mathrm{tr}}$, and the number of lattice sites $L=3 N_{\text {tr }} / 2$, so that the ground-state energy per site is identified as

$$
\varepsilon_{0}=\frac{2}{9}\left(\lambda_{\bar{A} \bar{A}}-2 \lambda_{A \bar{B}}+\lambda_{\bar{B} \bar{B}}\right)
$$

The parameters are related as

$$
\left[\begin{array}{l}
\lambda_{\bar{A} \bar{A}} \\
\lambda_{\bar{A} \bar{B}} \\
\lambda_{\bar{B} \bar{B}}
\end{array}\right]=\left[\begin{array}{ccc}
1 & 4 & 4 \\
1 & -2 & 1 \\
1 & 1 & -2
\end{array}\right]\left[\begin{array}{c}
U / 2 \\
W \\
X
\end{array}\right] .
$$


2

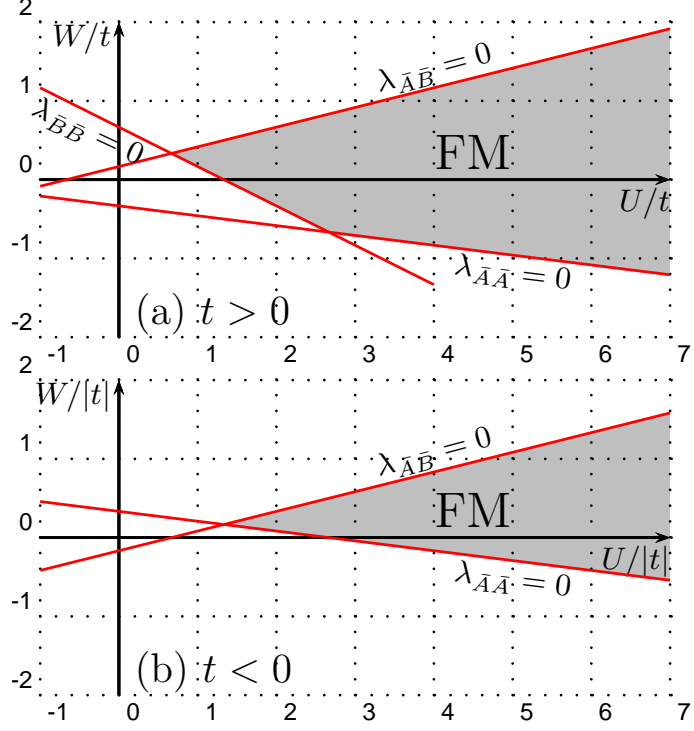

Fig. 8. Phase diagrams of the generalized Hubbard model on the Kagomé lattice, in the $U /|t|-W /|t|$ parameter space with $V_{\|}=W=W^{\prime}, V_{\perp}=U / 2, X=X^{\prime}=t / 3$. (a) and (b) correspond to the case of $t>0$ and $t<0$, respectively. The shaded regions labeled by FM denote the exact ferromagnetic ground state.

Then we have

$$
\begin{aligned}
& \lambda_{\bar{A} \bar{A}}=\frac{U}{2}+4 W+\frac{4}{3} t, \\
& \lambda_{\bar{A} \bar{B}}=\frac{U}{2}-2 W+\frac{t}{3} \\
& \lambda_{\bar{B} \bar{B}}=\frac{U}{2}+W-\frac{2}{3} t .
\end{aligned}
$$

Thus the condition of the exact ferromagnetic ground state is given as follows,

$$
\begin{aligned}
& W \geq-\frac{U}{8}-\frac{t}{3}, \quad W \leq \frac{U}{4}+\frac{t}{6}, \quad W \geq-\frac{U}{2}+\frac{2}{3} t \\
& V_{\perp}=\frac{U}{2}, \quad V_{\|}=W=W^{\prime}, \quad X=X^{\prime}=\frac{t}{3}
\end{aligned}
$$

The ground state energy per site is

$$
\varepsilon_{0}=2 W
$$

This is consistent with the fact that in the fully ferromagnetic state, only the $V_{\|}$term contribute to the energy. The condition of the hopping in Eq. (55) means that $t$ may take both positive and negative values. As shown in Fig 8 , (a) for positive $t$, the exact ferromagnetic ground state is surrounded by three lines, while (b) for the negative $t$, the lines become two.

In Fig. 9 the numerical results of the ground-state energy for the $1 / 2$-filling Kagomé model at $U /|t|=3$ are plotted as a function of $W /|t|$, where the numbers of spinup and spin-down electrons are kept to be as close as possible, namely, $N_{\uparrow}-N_{\downarrow}=0$ and $\left|N_{\uparrow}-N_{\downarrow}\right|=1$ for even- (a)

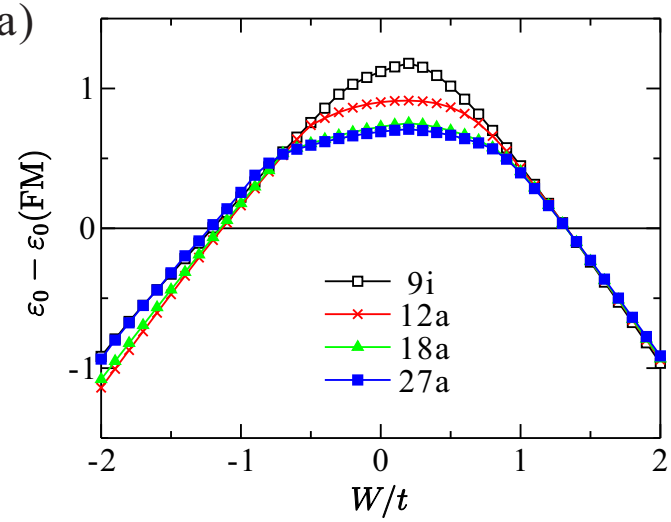

(b)

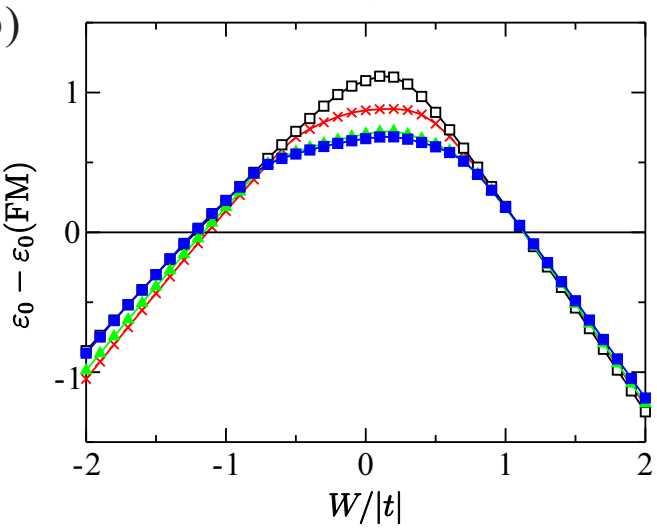

Fig. 9. Numerical results of the ground-state energy per site as a function of $W /|t|$ for the $1 / 2$-filling Kagomé model at $U /|t|=3$ with (a) $t>0$ and (b) $t<0$, where the number of spin-up and spin-down electrons are kept as $N_{\uparrow}=N_{\downarrow}$ or $N_{\uparrow}=N_{\downarrow}+1$. The energy of the FM state $\varepsilon_{0}(\mathrm{FM})=2 W$ is subtracted.

and odd-site clusters, respectively. Since the ground-state energy of the FM state $\varepsilon_{0}=2 W \equiv \varepsilon_{0}(\mathrm{FM})$ is subtracted in Fig. 9, the FM phase is indicated by a region having positive value of the numerical energy $\varepsilon_{0}-\varepsilon_{0}(\mathrm{FM})>0$. The finite-size effect seems to be much smaller than that in the PN state. For the both positive and negative $t$ values, the FM phase appears at $-1 \lesssim W /|t| \lesssim 1$, though the region for $t<0$ may be slightly narrower than that for $t>0$. The FM phase would be comparatively more extended than the analytical results shown in Fig 8 ,

\section{Entanglement entropy}

In this section we consider the entanglement entropy (EE) 20 of the system discussed above. When we divide the normalized wave function of the system into two regions $\mathrm{A}$ and $\mathrm{B}$ as

$$
|\Psi\rangle=\sum_{n m} \Lambda_{n m}\left|\Psi_{n}^{\mathrm{A}}\right\rangle \otimes\left|\Psi_{m}^{\mathrm{B}}\right\rangle,
$$

the $\mathrm{EE}$ is given by

$$
S^{\mathrm{A}}=-\operatorname{Tr}_{\mathrm{A}}\left[\hat{\rho}_{\mathrm{A}} \log \hat{\rho}_{\mathrm{A}}\right],
$$


(a)1D chain

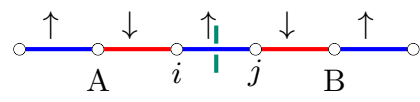

(b)Kagomé

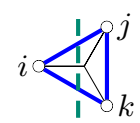

A I B

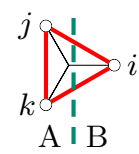

Fig. 10. Patterns to cut the systems into two regions $A$ and $\mathrm{B}$ to calculate the entanglement entropy (EE) for (a) the $1 \mathrm{D}$ chain and (b) the Kagomé lattice, respectively.

with the reduced density matrix

$$
\hat{\rho}_{\mathrm{A}}=\sum_{n m}\left(\Lambda \Lambda^{T}\right)_{n m}\left|\Psi_{n}^{\mathrm{A}}\right\rangle\left\langle\Psi_{m}^{\mathrm{A}}\right|,
$$

where $\Lambda^{T}$ is the transposed matrix of $\Lambda$.

For the BN state in 1D, $\left|\Psi_{n}^{\mathrm{A}}\right\rangle$ and $\left|\Psi_{n}^{\mathrm{B}}\right\rangle$ (see Fig. 10(a)) are given as

$$
\begin{aligned}
& \left|\Psi_{1}^{\mathrm{A}}\right\rangle=X_{\mathrm{A}}^{\dagger} c_{i \sigma}^{\dagger}|0\rangle_{\mathrm{A}}, \\
& \left|\Psi_{2}^{\mathrm{A}}\right\rangle=X_{\mathrm{A}}^{\dagger}|0\rangle_{\mathrm{A}}, \\
& \left|\Psi_{1}^{\mathrm{B}}\right\rangle=c_{j \sigma}^{\dagger} X_{\mathrm{B}}^{\dagger}|0\rangle_{\mathrm{B}}, \\
& \left|\Psi_{2}^{\mathrm{B}}\right\rangle=X_{\mathrm{B}}^{\dagger}|0\rangle_{\mathrm{B}},
\end{aligned}
$$

where $X_{\mathrm{A}}^{\dagger}$ and $X_{\mathrm{B}}^{\dagger}$ denote normalized operators that create the common parts of $\mathrm{A}$ and $\mathrm{B}$ regions, respectively. Then we get

$$
\Lambda=\frac{1}{\sqrt{2}}\left[\begin{array}{ll}
0 & 1 \\
1 & 0
\end{array}\right], \quad \Lambda \Lambda^{T}=\frac{1}{2}\left[\begin{array}{ll}
1 & 0 \\
0 & 1
\end{array}\right] .
$$

The EE is easily obtained by using the eigenvalues $\lambda_{i}$ of the matrix $\Lambda \Lambda^{T}$ as

$$
S^{\mathrm{A}}=-\sum_{i} \lambda_{i} \log \lambda_{i}=\log 2 .
$$

This result is for an open boundariy system where the two regions are cut at one bond. Therefore, the EE for the periodic boundariy system is $S^{\mathrm{A}}=\log 4$. These results can also be obtained by using the matrix product representation of the wave function [11,13.

For the PN state in the Kagomé lattice with $1 / 3$-filling, we consider a case that two regions $\mathrm{A}$ and $\mathrm{B}$ are connected via a single triangle, for simplicity, as shown in Fig. 10(b). Then $\left|\Psi_{n}^{\mathrm{A}}\right\rangle$ and $\left|\Psi_{n}^{\mathrm{B}}\right\rangle$ are given as

$$
\begin{aligned}
& \left|\Psi_{1}^{\mathrm{A}}\right\rangle=X_{\mathrm{A}}^{\dagger} c_{i \sigma}^{\dagger}|0\rangle_{\mathrm{A}}, \\
& \left|\Psi_{2}^{\mathrm{A}}\right\rangle=X_{\mathrm{A}}^{\dagger}|0\rangle_{\mathrm{A}}, \\
& \left|\Psi_{1}^{\mathrm{B}}\right\rangle=c_{j \sigma}^{\dagger} X_{\mathrm{B}}^{\dagger}|0\rangle_{\mathrm{B}}, \\
& \left|\Psi_{2}^{\mathrm{B}}\right\rangle=c_{k \sigma}^{\dagger} X_{\mathrm{B}}^{\dagger}|0\rangle_{\mathrm{B}}, \\
& \left|\Psi_{3}^{\mathrm{B}}\right\rangle=X_{\mathrm{B}}^{\dagger}|0\rangle_{\mathrm{B}} .
\end{aligned}
$$

In this case, we get the following matrix elements

$$
\Lambda=\frac{1}{\sqrt{3}}\left[\begin{array}{lll}
1 & 1 & 0 \\
0 & 0 & 1
\end{array}\right], \quad \Lambda \Lambda^{T}=\frac{1}{3}\left[\begin{array}{ll}
1 & 0 \\
0 & 2
\end{array}\right] .
$$

(a)

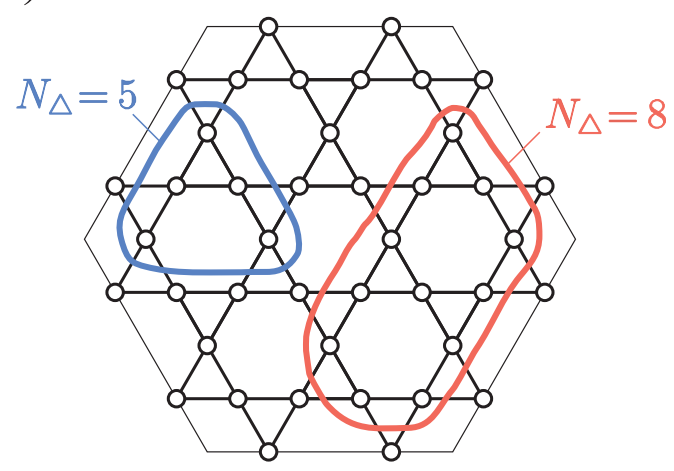

(b)

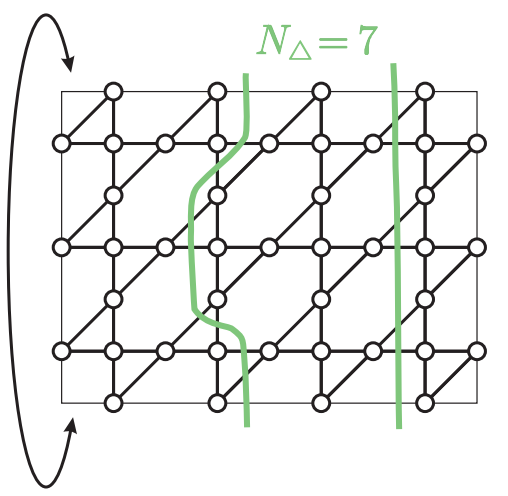

Fig. 11. (a) Isotropic periodic and (b) torus clusters of the Kagomé lattice. The bold (cutting) lines are examples of the system division.

If we cut the triangle in the opposite way, we should consider the situation $\mathrm{A} \leftrightarrow \mathrm{B}$. In this case the matrix in Eq. (65) becomes

$$
\Lambda^{T} \Lambda=\frac{1}{3}\left[\begin{array}{lll}
1 & 1 & 0 \\
1 & 1 & 0 \\
0 & 0 & 1
\end{array}\right]
$$

The eigenvalues of the matrix $\Lambda^{T} \Lambda$ are

$$
\lambda_{i}=\left\{\frac{1}{3}, \frac{2}{3}, 0\right\} .
$$

Thus the value of the EE does not depend on the ways to cut the triangle, so that we get the EE in general cases as

$$
S^{\mathrm{A}}=N_{\triangle} \underbrace{[\log 3-(2 / 3) \log 2]}_{s_{0}},
$$

where $s_{0}=0.636514168 \cdots$ and $N_{\triangle}$ means the number of triangles along the cutting lines. This means that the EE obeys the area law. The EE for the PN state at $2 / 3$ filling is obtained as the same value as that of $1 / 3$-filling via the particle-hole transformation. For the FM state at $1 / 2$ filling, the EE becomes zero.

The value of EE can be easily verified numerically by using the DMRG method. For the BN state in $1 \mathrm{D}$, the $\mathrm{EE}$ is $S^{\mathrm{A}}=1.38629437 \approx \log 4$ which does not depend 
on length of regions $\mathrm{A}$ and $\mathrm{B}$ in a periodic chain. For the PN state in 1/3-filled Kagomé lattice, some examples of the cutting lines are shown in Fig. 11. We obtain $S^{\mathrm{A}}=$ $3.182570841 \approx 5 s_{0}$ and $S^{\mathrm{A}}=5.092113346 \approx 8 s_{0}$ for the periodic cluster in Fig. 111(a); $S^{\mathrm{A}}=4.455599178 \approx 7 s_{0}$ for the torus cluster in Fig. 11(b). Thus, we have confirmed that the EE is proportional to the number of triangles on the cutting lines, i.e., $S^{\mathrm{A}}=N_{\triangle} s_{0}$.

\section{Summary and discussion}

In summary, we have discussed exact ground states of the generalized Hubbard model based on the projection operator method in multicomponent systems. The Hamiltonian with the exact ground state can be obtained when the lattices have bipartite structure in terms of corner sharing unit plaquettes. We have applied this method to the $1 \mathrm{D}$ chain and the Kagomé lattice, and obtained parameter regions of the exact ground states for several fillings. We have also calculated the entanglement entropy (EE). In addition, we have performed numerical calculations based on exact diagonalization and density-matrix renormalization group, and confirmed the results.

In the $1 \mathrm{D}$ chain, the exact ground state is the bond Néel (BN) state where the system has a Néel ordered state on the bonds 11,13 . This corresponds to the staggered dimer states in the spin-1/2 two-leg ladder model with four spin exchanges. 19] We have numerically confirmed the existence of the exact $\mathrm{BN}$ ground state. The $\mathrm{BN}$ phase may be expanded to the outside of the analytical argument. The ferromagnetic (FM) and BN phase boundary agrees perfectly between the analytical and numerical results.

In the Kagomé lattice, we have discussed the exact plaquette Néel (PN) state at 1/3-filling [17], and also the PN state at 2/3-filling as well as the FM state at halffilling. According to the numerical calculations, each the exact state seems to be stabilized in a wider region than those suggested by the analytical result. However, further calculations are required to corroborate it. For the EE, we have confirmed perfect agreement between the analytical and the numerical calculations.

In addition to the PN state, we may introduce other exact plaquette ground states. For example, the following state

$$
\left|\Psi_{\sigma}\right\rangle \equiv \prod_{\langle i j k\rangle \in \triangle} B_{i j k \sigma}^{\dagger} \prod_{\left\langle i^{\prime} j^{\prime} k^{\prime}\right\rangle \in \nabla} C_{i^{\prime} j^{\prime} k^{\prime} \bar{\sigma}}^{\dagger}|0\rangle,
$$

seems like a "topological state", since local spin current state with time-reversal symmetry [21]. In order to stabilize this state, we have to extend our model Hamiltonian to include the current terms $J_{i j k \sigma}$.

\section{Acknowledgment}

M. N. acknowledges the Visiting Researcher's Program of the Institute for Solid State Physics, the University of Tokyo, and the Max Planck Institute für Physik komplexer Systeme, Dresden where this work was initiated. M. N. is supported by JSPS KAKENHI Grant Number 17K05580. S. N. acknowledges support from the SFB 1143 of the Deutsche Forschungsgemeinschaft. S. N. would like to thank U. Nitzsche for technical assistance.

\section{References}

1. J. Hubbard, Proc. R. Soc. London A 276, 238 (1963); J. Kanamori, Prog. Theor. Phys. 30, 275 (1963); M. C. Gutzwiller, Phys. Rev. Lett. 10, 159 (1963)

2. D. K. Campbell, J. T. Gammel, and E. W. Loh, Jr., Phys. Rev. B 38, 12043 (1988); 42, 475 (1990)

3. M. E. Simón and A. A. Aligia, Phys. Rev. B 48, 7471 (1993)

4. R. Strack and D. Vollhardt, Phys. Rev. Lett. 70, 2637 (1993)

5. R. Strack and D. Vollhardt, Phys. Rev. Lett. 72, 3425 (1994)

6. L. Arrachea and A. A. Aligia, Phys. Rev. Lett. 73, 2240 (1994)

7. J. de Boer, V. E. Korepin, and A. Schadschneider, Phys. Rev. Lett. 74, 789 (1995)

8. J. de Boer and A. Schadschneider, Phys. Rev. Lett. 75, 4298 (1995)

9. A. Montorsi and D. K. Campbell, Phys. Rev. B 53, 5153 (1996)

10. M. Kollar, R. Strack, and D. Vollhardt, Phys. Rev. B 53, 9225 (1996)

11. K. Itoh, M. Nakamura, and N. Muramoto, J. Phys. Soc. Jpn. 70, 1202 (2001)

12. M. Nakamura and K. Itoh, J. Phys. Soc. Jpn. 70, 3606 (2001)

13. M. Nakamura, T. Okano and K. Itoh, Phys. Rev. B 72, 115121 (2005)

14. C. K. Majumder and D. K. Gohsh, J. Math. Phys. 10, 1388 (1969)

15. I. Affleck, T. Kennedy, E. Lieb, and H. Tasaki, Phys. Rev. Lett. 59, 799 (1987); Commun. Math. Phys. 115, 477 (1988)

16. K. Itoh, J. Phys. Soc. Jpn. 68, 322 (1999)

17. M. Nakamura and K. Itoh, J. Phys. Soc. Jpn. 74, 234 (2005)

18. S. R. White, Phys. Rev. Lett. 69, 2863 (1992)

19. A. K. Kolezhuk and H.-J. Mikeska, Phys. Rev. Lett. 80, 2709 (1998); Int. J. Mod. Phys. B 12, 2325 (1998)

20. For example, R. Horodecki, P. Horodecki, M. Horodecki, and K. Horodecki, Rev. Mod. Phys. 81, 865 (2009).

21. C. L. Kane and E. J. Mele, Phys. Rev. Lett. 95, 146802 (2005) 


\section{A Details of calculation}

For the 1D case, we have used the relation,

$T_{i j \uparrow} T_{i j \downarrow}=\underbrace{\frac{1}{2} \sum_{i j \sigma \sigma^{\prime}} T_{i j \sigma} T_{i j \sigma^{\prime}}}_{h_{W}}-\frac{1}{2} \sum_{\sigma}\left(n_{i \sigma}+n_{j \sigma}\right)+\underbrace{\sum_{\sigma} n_{i \sigma} n_{j \sigma}}_{h_{V_{\|}}}$.

For the Kagomé lattice, products of the operators generate the following terms,

$$
\begin{aligned}
& N_{i j k \uparrow} N_{i j k \downarrow}=\underbrace{n_{i \uparrow} n_{i \downarrow}+n_{j \uparrow} n_{j \downarrow}+n_{k \uparrow} n_{k \downarrow}}_{2 h_{U}} \\
& +\underbrace{n_{i \uparrow} n_{j \downarrow}+n_{j \uparrow} n_{k \downarrow}+n_{k \uparrow} n_{i \downarrow}+n_{j \uparrow} n_{i \downarrow}+n_{k \uparrow} n_{j \downarrow}+n_{i \uparrow} n_{k \downarrow}}_{h_{V_{\perp}}}, \\
& T_{i j k \uparrow} T_{i j k \downarrow}=\underbrace{\frac{1}{2} \sum_{\mu \nu \sigma \sigma^{\prime}} T_{\mu \nu \sigma} T_{\mu \nu \sigma^{\prime}}}_{h_{W}}-\sum_{\sigma} N_{i j k \sigma} \\
& +\underbrace{\sum_{\sigma}\left(n_{i \sigma} n_{j \sigma}+n_{j \sigma} n_{k \sigma}+n_{k \sigma} n_{i \sigma}\right)}_{h_{V_{\|}}} \\
& +\underbrace{\sum_{\sigma}\left(T_{i j \sigma} T_{j k \bar{\sigma}}+T_{j k \sigma} T_{k i \bar{\sigma}}+T_{k i \sigma} T_{i j \bar{\sigma}}\right)}_{h_{W^{\prime}}}, \\
& N_{i j k \uparrow} T_{i j k \downarrow}+T_{i j k \uparrow} N_{i j k \downarrow}=\underbrace{\sum_{\mu \nu \sigma} T_{\mu \nu \sigma}\left(n_{\mu \bar{\sigma}}+n_{\nu \bar{\sigma}}\right)}_{h_{X}} \\
& +\underbrace{\sum_{\sigma}\left(n_{i \sigma} T_{j k \bar{\sigma}}+n_{j \sigma} T_{k i \bar{\sigma}}+n_{k \sigma} T_{i j \bar{\sigma}}\right)}_{h_{X^{\prime}}}
\end{aligned}
$$

where $\sum_{\mu \nu}$ is taken for $\mu \nu \in\{i j, j k, k i\}$ in one triangle. The weight of $h_{U}$ term is doubled, because the on-site interaction is shared with the neighboring triangle.

\section{B Periodic Kagomé clusters used in DMRG calculations}

In the exact diagonalization and density-matrix renormalization group calculations for the Kagomé lattice, we used periodic clusters. The clusters shown in Figs. 12] and 13 are spatially anisotropic and isotropic, respectively. The periodicity of the plaquette Néel state is compatible with all the clusters.
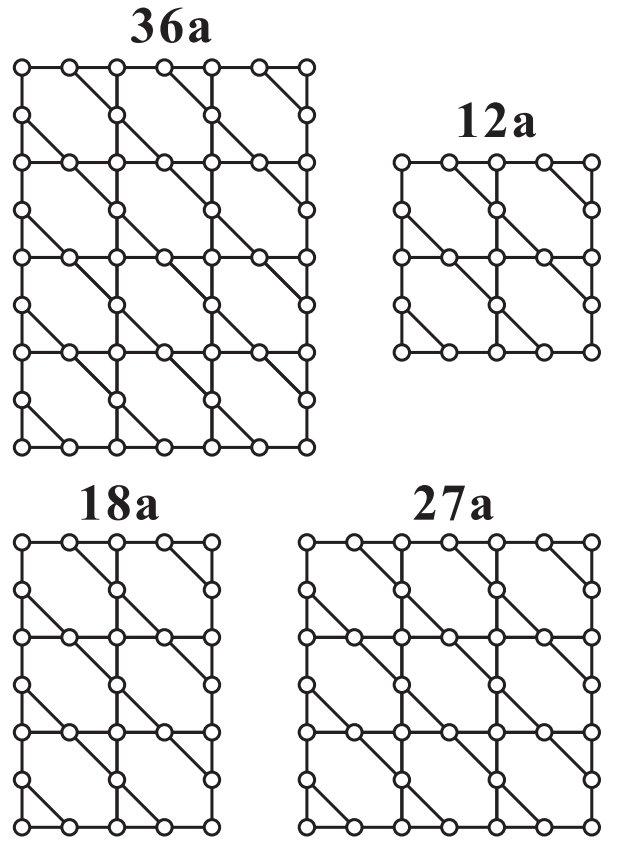

Fig. 12. Anisotropic Kagomé clusters.

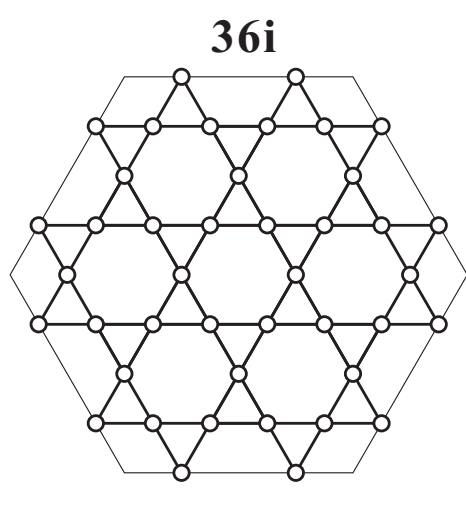

$27 \mathbf{i}$
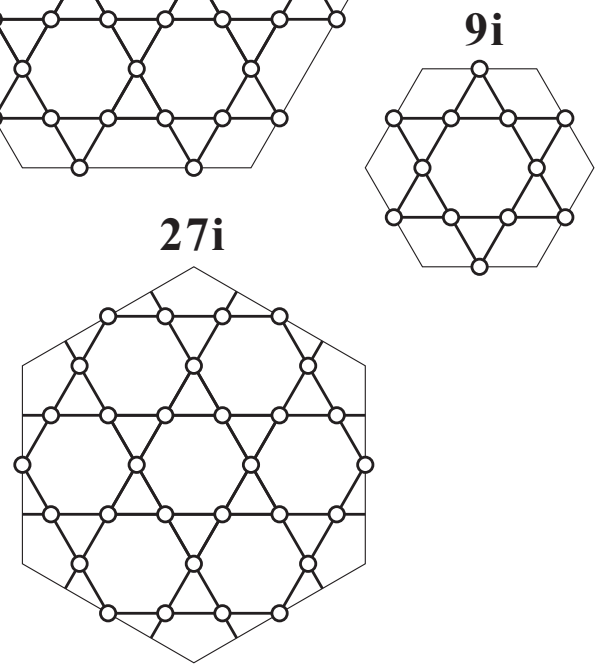

Fig. 13. Isotropic Kagomé clusters. 
